\title{
Pharmaceutical Quality/CMC Impurity Classification Terminology
}

National Cancer Institute

\section{Source}

National Cancer Institute. Pharmaceutical Quality/CMC Impurity Classification

Terminology. NCl Thesaurus. Code C133856.

A category of terminology used to qualify the information pertaining to impurity classification in the framework of the Pharmaceutical Quality/Chemistry, Manufacturing and Controls documents. 